\title{
On the intuitionistic fuzzy implication $\rightarrow_{191}$
}

\author{
Krassimir T. Atanassov \\ Institute of Biophysics and Biomedical Engineering \\ Bulgarian Academy of Sciences \\ Acad. G. Bonchev Str., Bl. 105, Sofia-1113, Bulgaria \\ e-mail: krat@bas.bg \\ and \\ Intelligent Systems Laboratory \\ "Prof. Asen Zlatarov" University \\ Burgas-8010, Bulgaria
}

Department of Bioinformatics and Mathematical Modelling

In memory of my friend Ivan Georgiev

Received: 4 October 2019

Accepted: 24 October 2019

Abstract: A new intutionistic fuzzy implication, $\rightarrow_{191}$, is constructed. Some of its basic properties are studied.

Keywords: Implication, Intuitionistic fuzzy implication, Intuitionistic fuzzy logic.

2010 Mathematics Subject Classification: 03E72.

\section{Introduction}

In [3], 185 intuituinistic fuzzy implications are defined. Five other intuituinistic fuzzy implications are introduced in [4-10]. Now a new intuituinistic fuzzy implication will be given.

In some definitions we shall use functions $\mathrm{sg}$ and $\overline{\mathrm{sg}}$ :

$$
\operatorname{sg}(x)=\left\{\begin{array}{cc}
1 & \text { if } x>0 \\
0 & \text { if } x \leq 0
\end{array}, \quad \overline{\operatorname{sg}}(x)=\left\{\begin{array}{cc}
0 & \text { if } x>0 \\
1 & \text { if } x \leq 0
\end{array}\right.\right.
$$


Let everywhere intuitionistic fuzzy truth values of variables $x$ and $y$ be

$$
x=\langle a, b\rangle, \quad y=\langle c, d\rangle .
$$

In [3], for the variables $x$ and $y$ operation "conjunction" $(\&)$ is defined by:

$$
V(x \& y)=\langle\min (a, c), \max (b, d)\rangle .
$$

The pair $\langle a, b\rangle$ is: a tautology if and only if (iff) $a=1$ and $b=0$, and is an intuitionistic fuzzy tautology (IFT) iff $a \geq b$.

\section{Main results}

Let us define

$$
x \rightarrow_{191} y=\langle a, b\rangle \rightarrow\langle c, d\rangle=\langle\overline{\operatorname{sg}}(a-c) \overline{\operatorname{sg}}(d-b), \operatorname{sg}(a-c) \operatorname{sg}(d-b)\rangle .
$$

For brevity, below we will write $\rightarrow$ instead of $\rightarrow_{191}$.

First, we will show that the definition of the new implication is correct. Let $a, b, c, d \in[0,1]$ such that $a+b \leq 1$ and $c+d \leq 1$. Then

$$
\begin{aligned}
& 0 \leq \overline{\operatorname{sg}}(a-c) \overline{\operatorname{sg}}(d-b) \leq 1, \\
& 0 \leq \operatorname{sg}(a-c) \operatorname{sg}(d-b) \leq 1,
\end{aligned}
$$

and if

$$
X \equiv \overline{\operatorname{sg}}(a-c) \overline{\operatorname{sg}}(d-b)+\operatorname{sg}(a-c) \operatorname{sg}(d-b),
$$

then, we obtain sequentially. If $a>c$, then $\operatorname{sg}(a-c)=1$ and $\overline{\operatorname{sg}}(a-c)=0$, i.e.,

$$
X=0+\operatorname{sg}(d-b) \leq 1 .
$$

If $a \leq c$, then, $\operatorname{sg}(a-c)=0$ and $\overline{\operatorname{sg}}(a-c)=1$, i.e., $X=\overline{\operatorname{sg}}(d-b) \leq 1$.

Therefore, the definition of the new implication is correct. It generates the following negation.

$$
\begin{array}{r}
\neg\langle a, b\rangle=\langle a, b\rangle \rightarrow\langle 0,1\rangle=\langle\overline{\operatorname{sg}}(a-0) \overline{\operatorname{sg}}(1-b), \operatorname{sg}(a-0) \operatorname{sg}(1-b)\rangle \\
=\langle\overline{\operatorname{sg}}(a) \overline{\operatorname{sg}}(1-b), \operatorname{sg}(a) \operatorname{sg}(1-b)\rangle=\langle\overline{\operatorname{sg}}(1-b), \operatorname{sg}(a)\rangle \\
= \begin{cases}\langle 1,0\rangle, & \text { if } a=0 \text { and } b=1 \\
\langle 0,0\rangle, & \text { if } a=0 \text { and } b<1 \\
& \text { or } a>0 \text { and } b=1 \\
\langle 0,1\rangle, & \text { if } a>0 \text { and } b<1\end{cases}
\end{array}
$$

Theorem 1. The new implication $\rightarrow_{191}$ :

(a) satisfies $x \rightarrow x$ as a tautology;

(b) satisfies $x \rightarrow \neg \neg x$ as a tautology; 
(c) does not satisfy $\neg \neg x \rightarrow x$ even as an IFT.

Proof. First, we see that

$$
\begin{aligned}
& \overline{\operatorname{sg}}(1-\operatorname{sg}(a))=\left\{\begin{array}{l}
\text { if } a=0: \overline{\operatorname{sg}}(1)=0 \\
\text { if } a>0: \overline{\operatorname{sg}}(0)=1
\end{array}=\operatorname{sg}(a),\right. \\
& \operatorname{sg}(\overline{\operatorname{sg}}(1-b))=\left\{\begin{array}{l}
\text { if } b=1: \operatorname{sg}(1)=1 \\
\text { if } b<1: \operatorname{sg}(0)=0
\end{array}=\overline{\operatorname{sg}}(1-b),\right. \\
& \overline{\operatorname{sg}}(a-\operatorname{sg}(a))=\left\{\begin{array}{l}
\text { if } a=0: \overline{\operatorname{sg}}(0)=1 \\
\text { if } a>0: \overline{\operatorname{sg}}(0)=1
\end{array}=1,\right. \\
& \operatorname{sg}(a-\operatorname{sg}(a))=\left\{\begin{array}{l}
\text { if } a=0: \operatorname{sg}(0)=0 \\
\text { if } a>0: \operatorname{sg}(a-1)=0
\end{array}=0,\right. \\
& \overline{\operatorname{sg}}(\overline{\operatorname{sg}}(1-b)-b)=\left\{\begin{array}{l}
\text { if } b=1: \overline{\operatorname{sg}}(0)=1 \\
\text { if } b<1: \overline{\operatorname{sg}}(0)=1
\end{array}=1,\right. \\
& \overline{\operatorname{sg}}(\operatorname{sg}(a)-a)=\left\{\begin{array}{l}
\text { if } a=0: \overline{\operatorname{sg}}(0)=1 \\
\text { if } a=0: \overline{\operatorname{sg}}(0)=1 \\
\text { if } 0<a<1: \overline{\operatorname{sg}}(1)=0
\end{array},\right. \\
& \overline{\operatorname{sg}}(b-\overline{\operatorname{sg}}(1-b))=\left\{\begin{array}{l}
\text { if } b=0: \overline{\operatorname{sg}}(0)=1 \\
\text { if } b=1: \overline{\operatorname{sg}}(0)=1 \\
\text { if } 0<b<1: \overline{\operatorname{sg}}(1)=0
\end{array},\right.
\end{aligned}
$$

Then

$$
\neg \neg\langle a, b\rangle=\neg\langle\overline{\operatorname{sg}}(1-b), \operatorname{sg}(a)\rangle=\langle\overline{\operatorname{sg}}(1-\operatorname{sg}(a)), \operatorname{sg}(\overline{\operatorname{sg}}(1-b))\rangle
$$

For (a) we obtain:

$$
x \rightarrow x=\langle a, b\rangle \rightarrow\langle a, b\rangle=\langle\overline{\operatorname{sg}}(0) \overline{\operatorname{sg}}(0), \operatorname{sg}(0) \operatorname{sg}(0)\rangle=\langle 1,0\rangle .
$$

For (b) we obtain:

$$
\begin{gathered}
x \rightarrow \neg \neg x=\langle a, b\rangle \rightarrow \neg \neg\langle a, b\rangle \\
=\langle a, b\rangle \rightarrow\langle\overline{\operatorname{sg}}(1-\operatorname{sg}(a)), \operatorname{sg}(\overline{\operatorname{sg}}(1-b))\rangle \\
=\langle\overline{\operatorname{sg}}(a-\operatorname{sg}(a)) \overline{\operatorname{sg}}(\overline{\operatorname{sg}}(1-b)-b), \operatorname{sg}(a-\operatorname{sg}(a)) \operatorname{sg}(\overline{\operatorname{sg}}(1-b)-b)\rangle \\
=\langle\overline{\operatorname{sg}}(\overline{\operatorname{sg}}(1-b)-b), 0 \cdot \operatorname{sg}(\overline{\operatorname{sg}}(1-b)-b)\rangle=\langle 1,0\rangle .
\end{gathered}
$$

For (c) we see directly that

$$
\begin{gathered}
\neg \neg x \rightarrow x=\neg \neg\langle a, b\rangle \rightarrow\langle a, b\rangle \\
=\langle\overline{\operatorname{sg}}(1-\operatorname{sg}(a)), \operatorname{sg}(\overline{\operatorname{sg}}(1-b))\rangle \rightarrow\langle a, b\rangle
\end{gathered}
$$

$=\langle\overline{\operatorname{sg}}(\overline{\operatorname{sg}}(1-\operatorname{sg}(a))-a) \overline{\operatorname{sg}}(b-\operatorname{sg}(\overline{\operatorname{sg}}(1-b))), \operatorname{sg}(\overline{\operatorname{sg}}(1-\operatorname{sg}(a))-a) \operatorname{sg}(b-\operatorname{sg}(\overline{\operatorname{sg}}(1-b)))\rangle$ $=\langle\overline{\operatorname{sg}}(\overline{\operatorname{sg}}(1-\operatorname{sg}(a))-a) \overline{\operatorname{sg}}(b-\operatorname{sg}(\overline{\operatorname{sg}}(1-b))), \operatorname{sg}(\overline{\operatorname{sg}}(1-\operatorname{sg}(a))-a) \operatorname{sg}(b-\operatorname{sg}(\overline{\operatorname{sg}}(1-b)))\rangle$ 
(for $0<a, b<1$ )

$$
=\langle 0,1\rangle
$$

i.e., it is not an IFT.

Theorem 2. The new implication $\rightarrow_{191}$ :

(a) satisfies Modus Ponens in tautological sense,

(b) does not satisfy Modus Ponens in the IFT-sense,

(c) satisfies for every two variables $x$ and $y$,

$$
(x \&(x \rightarrow y)) \rightarrow y
$$

in the IFT-sense.

Proof. (a) Let $\langle a, b\rangle$ be a tautology, i.e., $a=1$ and $b=0$ and let $\langle a, b\rangle \rightarrow\langle c, d\rangle$ be a tautology, i.e., $\langle\overline{\operatorname{sg}}(a-c) \overline{\operatorname{sg}}(d-b)=1$ and $\operatorname{sg}(a-c) \operatorname{sg}(d-b)=0$. Then $1=\overline{\operatorname{sg}}(1-c) \overline{\operatorname{sg}}(d-0)=\overline{\operatorname{sg}}(1-c) \overline{\operatorname{sg}}(d)$, i.e. $1=\overline{\operatorname{sg}}(1-c)$ and hence $c=1$ and $d=0$. Therefore $\langle c, d\rangle$ is a tautology.

(b) Let $\langle a, b\rangle=\langle 0,0\rangle$, i.e., an IFT. Then, $\langle\overline{\operatorname{sg}}(a-c) \overline{\operatorname{sg}}(d-b), \operatorname{sg}(a-c) \operatorname{sg}(d-b)\rangle$ will be an IFT, e.g., for $\langle c, d\rangle=\langle 0.1,0.2\rangle$, but the last pair is not an IFT.

(c) we have sequentially:

$$
\begin{gathered}
V((x \&(x \rightarrow y)) \rightarrow y) \\
=(\langle a, b\rangle \&\langle\overline{\operatorname{sg}}(a-c) \overline{\operatorname{sg}}(d-b), \operatorname{sg}(a-c) \operatorname{sg}(d-b)\rangle) \rightarrow\langle c, d\rangle \\
=\langle\min (a, \overline{\operatorname{sg}}(a-c) \overline{\operatorname{sg}}(d-b)), \max (b, \operatorname{sg}(a-c) \operatorname{sg}(d-b))\rangle \rightarrow\langle c, d\rangle \\
=\langle\overline{\operatorname{sg}}(\min (a, \overline{\operatorname{sg}}(a-c) \overline{\operatorname{sg}}(d-b))-c) \overline{\operatorname{sg}}(d-\max (b, \operatorname{sg}(a-c) \operatorname{sg}(d-b)), \\
\operatorname{sg}(\min (a, \overline{\operatorname{sg}}(a-c) \overline{\operatorname{sg}}(d-b))-c) \operatorname{sg}(d-\max (b, \operatorname{sg}(a-c) \operatorname{sg}(d-b)))\rangle .
\end{gathered}
$$

Let

$$
\begin{aligned}
& X \equiv \overline{\operatorname{sg}}(\min (a, \overline{\operatorname{sg}}(a-c) \overline{\operatorname{sg}}(d-b))-c) \overline{\operatorname{sg}}(d-\max (b, \operatorname{sg}(a-c) \operatorname{sg}(d-b)) \\
& -\operatorname{sg}(\min (a, \overline{\operatorname{sg}}(a-c) \overline{\operatorname{sg}}(d-b))-c) \operatorname{sg}(d-\max (b, \operatorname{sg}(a-c) \operatorname{sg}(d-b)))
\end{aligned}
$$

If $a>c$, then

$$
\begin{gathered}
X=\overline{\operatorname{sg}}(\min (a, 0)-c) \overline{\operatorname{sg}}(d-\max (b, \operatorname{sg}(d-b))-\operatorname{sg}(\min (a, 0)-c) \operatorname{sg}(d-\max (b, \operatorname{sg}(d-b)) \\
=\overline{\operatorname{sg}}(-c) \overline{\operatorname{sg}}(d-\max (b, \operatorname{sg}(d-b))-\operatorname{sg}(-c) \operatorname{sg}(d-\max (b, \operatorname{sg}(d-b))) \\
=\overline{\operatorname{sg}}(d-\max (b, \operatorname{sg}(d-b)) \geq 0 .
\end{gathered}
$$

If $a \leq c$, then

$X=\overline{\operatorname{sg}}(\min (a, \overline{\operatorname{sg}}(d-b))-c) \overline{\operatorname{sg}}(d-\max (b, 0))-\operatorname{sg}(\min (a, \overline{\operatorname{sg}}(d-b))-c) \operatorname{sg}(d-\max (b, 0))$

$$
=\overline{\operatorname{sg}}(\min (a, \overline{\operatorname{sg}}(d-b))-c) \overline{\operatorname{sg}}(d-b))-\operatorname{sg}(\min (a, \overline{\operatorname{sg}}(d-b))-c) \operatorname{sg}(d-b) .
$$


If $d>b$, then

$$
X=\overline{\operatorname{sg}}(\min (a, 0)-c) \cdot 0-\operatorname{sg}(\min (a, 1)-c)=0-\operatorname{sg}(a-c)=0 .
$$

If $d \leq b$, then

$$
X=\overline{\operatorname{sg}}(\min (a, 1)-c)-\operatorname{sg}(\min (a, 1)-c) \cdot 0=\overline{\operatorname{sg}}(a-c)=1,
$$

i.e., the expression is an IFT.

Following [3], let us define for $x$

$$
\square x=\langle a, 1-a\rangle .
$$

Theorem 3. The new implication $\rightarrow_{191}$ satisfies the formula

$$
\square(x \rightarrow y) \rightarrow(\square x \rightarrow \square y)
$$

as a tautology.

Proof. We obtain sequentially

$$
\begin{gathered}
\square(x \rightarrow y) \rightarrow(\square x \rightarrow \square y) \\
=\square(\langle a, b\rangle \rightarrow\langle c, d\rangle) \rightarrow(\square\langle a, b\rangle \rightarrow \square\langle c, d\rangle) \\
=\square(\langle\overline{\operatorname{sg}}(a-c) \overline{\operatorname{sg}}(d-b), \operatorname{sg}(a-c) \operatorname{sg}(d-b)\rangle) \rightarrow(\langle a, 1-a\rangle \rightarrow\langle c, 1-c\rangle) \\
=\langle\overline{\operatorname{sg}}(a-c) \overline{\operatorname{sg}}(d-b), 1-\overline{\operatorname{sg}}(a-c) \overline{\operatorname{sg}}(d-b)\rangle \rightarrow\langle\overline{\operatorname{sg}}(a-c) \overline{\operatorname{sg}}(1-c-1+a), \operatorname{sg}(a-c) \operatorname{sg}(1-c-1+a)\rangle \\
=\langle\overline{\operatorname{sg}}(a-c) \overline{\operatorname{sg}}(d-b), 1-\overline{\operatorname{sg}}(a-c) \overline{\operatorname{sg}}(d-b)\rangle \rightarrow\langle\overline{\operatorname{sg}}(a-c) \overline{\operatorname{sg}}(a-c), \operatorname{sg}(a-c) \operatorname{sg}(a-c)\rangle
\end{gathered}
$$

(because $\overline{\operatorname{sg}}(p) \overline{\operatorname{sg}}(p)=\overline{\operatorname{sg}}(p)$ and $\operatorname{sg}(p) \operatorname{sg}(p)=\operatorname{sg}(p)$ for each $p \in[0,1]$ )

$$
\begin{gathered}
=\langle\overline{\operatorname{sg}}(a-c) \overline{\operatorname{sg}}(d-b), 1-\overline{\operatorname{sg}}(a-c) \overline{\operatorname{sg}}(d-b)\rangle \rightarrow\langle\overline{\operatorname{sg}}(a-c), \operatorname{sg}(a-c)\rangle \\
=\langle\overline{\operatorname{sg}}(\overline{\operatorname{sg}}(a-c) \overline{\operatorname{sg}}(d-b)-\overline{\operatorname{sg}}(a-c)) \overline{\operatorname{sg}}(\operatorname{sg}(a-c)-1+\overline{\operatorname{sg}}(a-c) \overline{\operatorname{sg}}(d-b)), \\
\operatorname{sg}(\overline{\operatorname{sg}}(a-c) \overline{\operatorname{sg}}(d-b)-\overline{\operatorname{sg}}(a-c)) \operatorname{sg}(\operatorname{sg}(a-c)-1+\overline{\operatorname{sg}}(a-c) \overline{\operatorname{sg}}(d-b))\rangle .
\end{gathered}
$$

Let

$$
\begin{gathered}
X=\langle\overline{\operatorname{sg}}(\overline{\operatorname{sg}}(a-c) \overline{\operatorname{sg}}(d-b)-\overline{\operatorname{sg}}(a-c)) \overline{\operatorname{sg}}(\operatorname{sg}(a-c)-1+\overline{\operatorname{sg}}(a-c) \overline{\operatorname{sg}}(d-b)), \\
\operatorname{sg}(\overline{\operatorname{sg}}(a-c) \overline{\operatorname{sg}}(d-b)-\overline{\operatorname{sg}}(a-c)) \operatorname{sg}(\operatorname{sg}(a-c)-1+\overline{\operatorname{sg}}(a-c) \overline{\operatorname{sg}}(d-b))\rangle .
\end{gathered}
$$

If $a>c$, then

$$
\begin{gathered}
x=\langle\overline{\operatorname{sg}}(0 \cdot \overline{\operatorname{sg}}(d-b)-0) \overline{\operatorname{sg}}(1-1+0 \cdot \overline{\operatorname{sg}}(d-b)), \operatorname{sg}(0 \cdot \overline{\operatorname{sg}}(d-b)-0)) \operatorname{sg}(1-1+\overline{\operatorname{sg}}(d-b))\rangle \\
=\langle\overline{\operatorname{sg}}(0) \overline{\operatorname{sg}}(0), \operatorname{sg}(0) \operatorname{sg}(\overline{\operatorname{sg}}(d-b))\rangle=\langle 1,0\rangle .
\end{gathered}
$$

If $a \leq c$, then

$$
X=\langle\overline{\operatorname{sg}}(\overline{\operatorname{sg}}(d-b)-1) \overline{\operatorname{sg}}(-1+\overline{\operatorname{sg}}(d-b)), \operatorname{sg}(\overline{\operatorname{sg}}(d-b)-1) \operatorname{sg}(-1+\overline{\operatorname{sg}}(d-b))\rangle
$$

(because $\overline{\operatorname{sg}}(d-b) \leq 1$ )

$$
X=\langle\overline{\operatorname{sg}}(0), \operatorname{sg}(\overline{\operatorname{sg}}(d-b)-1)\rangle=\langle 1,0\rangle .
$$

Therefore formula $(*)$ is a tautology. Hence, it is an IFT, too. 


\section{Conclusion}

In this paper we have introduced the 191-st intuitionistic fuzzy implication, and have shown that it has intuitionistic but not classical behaviour. Several of its properties were formulated and proved. In a future research, new properties of the new implications will be studied.

Open Problem: In [2] six Cartesian products are defined (the last of them was published in [1]). For which index $j$ the $j$-th Cartesian product satisfies equality

$$
\left(A \rightarrow_{191} B\right) \times{ }_{j} C=\left(A \times{ }_{j} C\right) \rightarrow_{191}\left(B \times{ }_{j} C\right)
$$

for every three IFSs $A, B, C$ ?

\section{References}

[1] Andonov, V. (2008). On some properties of one Cartesian product over intuitionistic fuzzy sets. Notes on Intuitionistic Fuzzy Sets, 14 (1), 12-19.

[2] Atanassov, K. (2012). On Intuitionistic Fuzzy Sets Theory, Springer, Berlin.

[3] Atanassov K. (2017). Intuitionistic Fuzzy Logics. Springer, Cham, 2017.

[4] Atanassov, K., Angelova, N., Szmidt, E, \& Kacprzyk, J. (2016). Properties of the intuitionistic fuzzy implication $\rightarrow_{186}$. Notes on Intuitionistic Fuzzy Sets, 22 (4), 6-12.

[5] Atanassov, K., Ribagin, S., Doukovska, L. \& Atanassova, V. (2017). Intuitionistic fuzzy implication $\rightarrow_{190}$. Notes on Intuitionistic Fuzzy Sets, 23 (4), 79-83.

[6] Atanassov, K., Szmidt, E. \& Kacprzyk, J. (2016). New Fodor's type of intuitionistic fuzzy implication and negation. Notes on Intuitionistic Fuzzy Sets, 22 (3), 1-8.

[7] Atanassov, K., Szmidt, E. \& Kacprzyk, J. (2017). Intuitionistic fuzzy implication $\rightarrow_{188}$. Notes on Intuitionistic Fuzzy Sets, 23 (1), 6-13.

[8] Atanassov, K., Szmidt, E. \& Kacprzyk, J. (2017). Intuitionistic fuzzy implication $\rightarrow_{187}$. Notes on Intuitionistic Fuzzy Sets, 23 (2), 37-43.

[9] Atanassov, K., Szmidt, E. \& Angelova, N. (2017). Properties of the intuitionistic fuzzy implication $\rightarrow_{187}$. Notes on Intuitionistic Fuzzy Sets, 23 (3), 3-8.

[10] Atanassova, L. (2017). Intuitionistic fuzzy implication $\rightarrow_{189}$. Notes on Intuitionistic Fuzzy Sets, 23 (1), 14-20. 